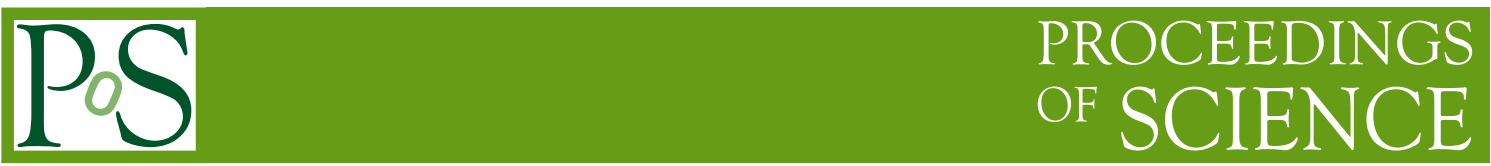

\title{
Status and Physics of the SHiP experiment at CERN
}

\author{
Annarita Buonaura*† \\ Universitá di Napoli Federico II e INFN Napoli \\ E-mail: annarita.buonaura@cern.ch
}

\begin{abstract}
SHIP is a new general purpose fixed target facility, whose Technical Proposal has been recently reviewed by the CERN SPS Committee and by the CERN Research Board. The two boards recommended that the experiment proceeds further to a Comprehensive Design phase in the context of the new CERN Working group "Physics Beyond Colliders", aiming at presenting a CERN strategy for the European Strategy meeting of 2019. In its initial phase, the $400 \mathrm{GeV}$ proton beam extracted from the SPS will be dumped on a heavy target with the aim of integrating $2 \times 10^{20}$ pot in 5 years. A dedicated detector, based on a long vacuum tank followed by a spectrometer and particle identification detectors, will allow probing a variety of models with light long-lived exotic particles and masses below $\mathrm{O}(10) \mathrm{GeV} / \mathrm{c}^{2}$. The main focus will be the physics of the socalled Hidden Portals, i.e. search for Dark Photons, Light scalars and pseudo-scalars, and Heavy Neutrinos. The sensitivity to Heavy Neutrinos will allow for the first time to probe, in the mass range between the kaon and the charm meson mass, a coupling range for which Baryogenesis and active neutrino masses could also be explained. Another dedicated detector will allow the study of neutrino cross-sections and angular distributions. $v_{\tau}$ deep inelastic scattering cross sections will be measured with a statistics 1000 times larger than currently available, with the extraction of the $F_{4}$ and $F_{5}$ structure functions, never measured so far and allow for new tests of lepton non-universality with sensitivity to BSM physics.
\end{abstract}

XXV International Workshop on Deep-Inelastic Scattering and Related Subjects

3-7 April 2017

University of Birmingham, $U K$

\footnotetext{
* Speaker.

${ }^{\dagger}$ On behalf of the SHiP Collaboration.
} 


\section{Introduction}

The world of particle physics is currently described by the Standard Model, which was ultimately validated after the discovery of the Higgs Boson by the ATLAS and CMS collaborations ( [1], [2]) in 2012.

However, its inability in explaining the origin of neutrino masses or of the baryon asymmetry or the presence of non-baryonic dark matter, tells us that the SM cannot be considered a complete theory: there must be new physics, even though its nature and its energy scale are still unknown. Two complementary approaches can be followed to search for the yet unknown particles or interactions that could solve the problems of the SM: it is either possible to go at always increasing energies (the so-called energy frontier research) or to focus on very weakly couplings (the so-called intensity frontier research).

SHiP (Search for Hidden Particles) is a new experiment proposed at the CERN SPS Committee in 2015 with the submission of a Technical Proposal [3] and a Physics Paper [4]. It aims at the observation of long lived very weakly interacting particles (Hidden Particles, HP) and can thus be included within the experiments that explore the intensity frontier, hence offering a complementary new physics search program complementary to that of LHC. Thanks to the peculiar properties of the facility, the SHiP experiment can also be considered a SM neutrino factory, in particular of tau neutrinos. Hence, it is designed to host both a Hidden Particle detector and a dedicated detector to study active neutrino cross-sections.

\section{The SHiP experiment}

The SHiP experiment is a general fixed target facility using $400 \mathrm{GeV}$ protons from the SPS which will impinge on a hybrid target made of blocks of titanium-zirconium doped molybdenum (TZM) followed by blocks of pure tungsten. In five years of data taking, it will collect $2 \times 10^{20}$ protons on target (p.o.t.).

The energy of $400 \mathrm{GeV}(\sqrt{s}=27 \mathrm{GeV})$ is optimal to maximise the production of charmed hadrons because at this energy the production cross sections of $c \bar{c}$ tend to saturate (fig.1b) [5-7].

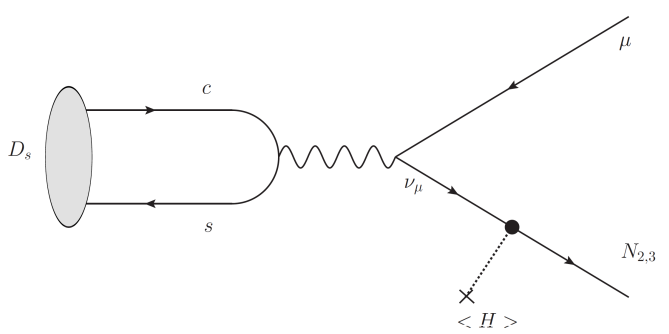

(a) Example of a charmed hadron decay producing Heavy Neutral Lepton.

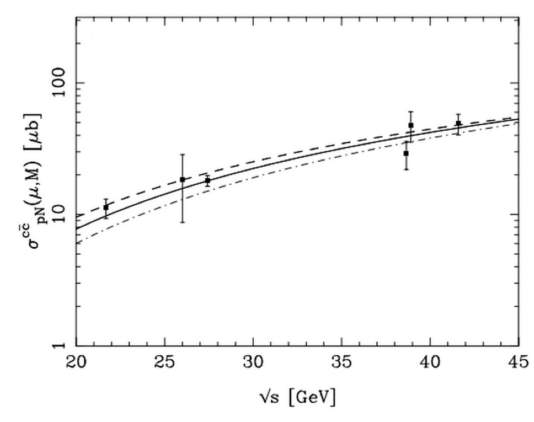

(b) Total cī production cross sections at fixed target energies [5], [6], [7].

Figure 1: Example of a production mechanism of HNLs and $c \bar{c}$ cross-section for different $\sqrt{s}$ 
Choosing target materials with a short interaction length allows to maximize neutrinos from charmed hadrons, to which the Hidden Particles (HP) couple, while minimising those coming from pions and kaons. The length of the target is also optimised to contain possible hadronic showers with minimum leakage. To have a greater efficiency, a hadron stopper made of $5 \mathrm{~m}$ of Iron is placed downstream of the target.

HP are supposed to have a long life-time with a decay length of $\mathrm{O}(\mathrm{km})$ and are hypothesised to decay in SM particles. To detect their decay products, a $50 \mathrm{~m}$ long decay vessel, equipped with detectors at the far end, is needed. The positioning of the decay volume with respect to the proton target is mainly driven by the large transverse momentum shown by the HP which are produced in the decay of charmed hadrons. Therefore, to optimise the HP acceptance it is better to place the decay volume as close as possible to the proton target.

Since SHiP aims at looking at very rare events, it must be a zero background experiment. The main source of background is given by the muon flux coming from short-lived resonances and from the decay of residual pions and kaons. To reduce this type of background, an active muon shield based on magnetic deflection of the muons in the horizontal plane is introduced right after the hadron stopper. The current configuration, still in process of optimisation in view of the Comprehensive Design Study to be handed in by the end of 2018, uses a muon shield $28 \mathrm{~m}$ long and magnetises also the $5 \mathrm{~m}$ of hadron stopper. The decay vessel is shaped as an elliptical frustum so to be fully contained in the region cleared from the muon background. The entrance window of the vessel is an ellipse with a major axis of $4 \mathrm{~m}$ and a minor axis of $2 \mathrm{~m}$. The rear window of the vessel is again an ellipse, but this time the major axis is $10 \mathrm{~m}$ long and the minor axis is $5 \mathrm{~m}$ long.

Other backgrounds are the combinatorial background from the residual muons entering the decay volume, muons deflecting off the cavern walls and SM neutrinos which can interact in the material upstream of the fiducial volume (the vessel is kept at low pressure $\left(10^{-6}-10^{-5}\right.$ bar $)$ with subsequent production of charged and neutral particles. To prevent them, the proton spills are prepared with a slow (1s) and very uniform beam extraction and a combination of light taggers is located upstream and at the beginning of the fiducial volume.

The current setup of the SHiP experiment is shown in fig. 2.

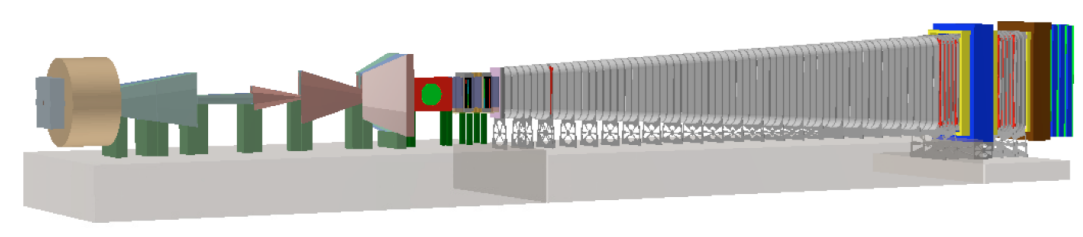

Figure 2: New layout of the SHiP experiment with a shorter muon shield and an elliptical frustum shaped decay vessel. 


\section{The Neutrino Detector}

The Neutrino Detector is designed to perform the first direct observation of the $\bar{v}_{\tau}$ and, thanks to the expected unprecedented statistics of both tau neutrinos and anti-neutrinos, also to study their properties and their cross-section. As a matter of fact, with a $400 \mathrm{GeV}$ proton beam, the production of charmed mesons and in particular of $D_{s}$ mesons is enhanced, thus resulting in a high flux of $v_{\tau}$ and $\bar{v}_{\tau}$, together with neutrinos of all flavours.

The Neutrino Detector is located in between the active muon shield and the decay vessel. It is placed in a horizontal magnetic field and it exploits the Emulsion Cloud Chamber Technology. The use of emulsion films as tracking devices guarantees the position resolution of $\mathrm{O}(\mu \mathrm{m})$ needed to disentangle the tau lepton decay vertex from the neutrino interaction vertex. This peculiarity, together with the high electron identification efficiency, makes this detector also suitable to perform dark sector exploration (e.g. look for sub-GeV Dark Matter produced in the decay of the dark photon) through the scattering of hidden particles with the electrons in the emulsion target.

The main unit of the Neutrino Detector is the brick (lead plates interleaved with emulsion films) followed downstream by a Compact Emulsion Spectrometer (CES, 3 emulsion films interleaved with light material) needed to measure the charge and momentum of hadrons produced in neutrino interactions and short lived particles decays. A Muon Magnetic Spectrometer is placed immediately downstream to measure the charge and the momentum of muons produced in charged current muon neutrino interactions or in $\tau \rightarrow \mu$ decays.

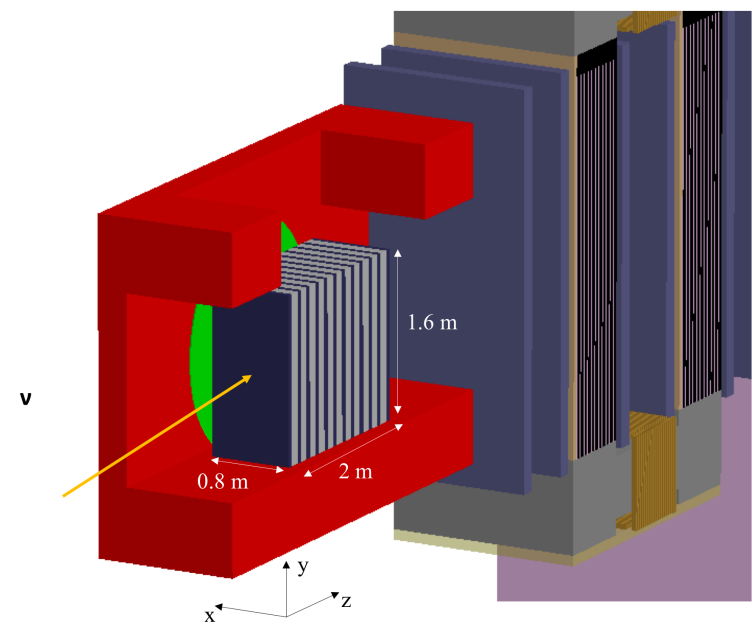

(a) Layout of the neutrino detector.

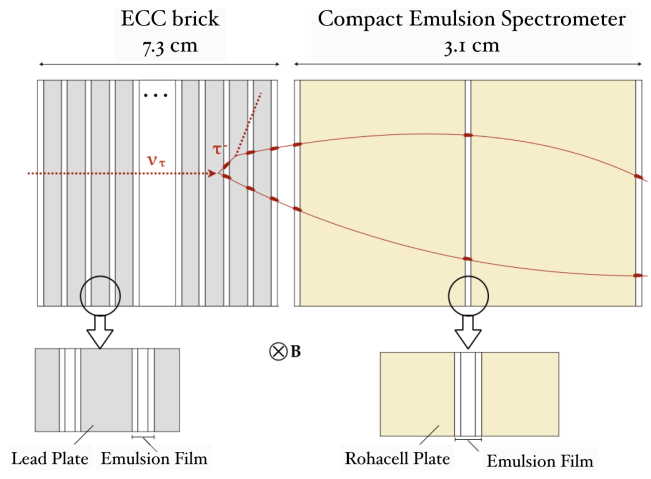

(b) Schematic representation of the neutrino detector unitary cell.

Figure 3: View of the tau neutrino detector and of its fundamental unit.

\subsection{Physics with the Neutrino Detector}

Tau neutrinos are copiously produced via $D_{s} \rightarrow \tau \nu_{\tau}$ and subsequent $\tau \rightarrow \nu_{\tau}$ decay. In five years running, $2.2 \times 10^{16} v_{\tau}+\bar{v}_{\tau}$ per $2 \times 10^{20}$ protons on target are expected. A high rate of $v_{e}$ and $v_{\mu}$, induced by the decay of soft pions and kaons, is also foreseen. The expected number of CC deep-inelastic neutrino interactions during the whole data taking is reported in the right column 


\begin{tabular}{ccccccc}
\hline $\begin{array}{c}\text { Neutrino } \\
\text { flavour }\end{array}$ & $\begin{array}{c}<\mathrm{E}> \\
\mathrm{GeV}\end{array}$ & $\begin{array}{c}\text { Beam } \\
\text { Dump }\end{array}$ & $\begin{array}{c}<\mathrm{E}> \\
\mathrm{GeV}\end{array}$ & $\begin{array}{c}\text { Neutrino } \\
\text { Detector }\end{array}$ & $\begin{array}{c}<\mathrm{E}> \\
\mathrm{GeV}\end{array}$ & $\begin{array}{c}\mathrm{CC} \\
\mathrm{DIS}\end{array}$ \\
\hline$v_{\mu}$ & 1.5 & $3.4 \times 10^{18}$ & 10.7 & $4.4 \times 10^{16}$ & 31 & $2.3 \times 10^{6}$ \\
$v_{e}$ & 4.1 & $2.2 \times 10^{17}$ & 29.5 & $5.7 \times 10^{15}$ & 50 & $6.3 \times 10^{5}$ \\
$v_{\tau}$ & 7.4 & $1.1 \times 10^{16}$ & 22.3 & $4.7 \times 10^{14}$ & 45 & $2.6 \times 10^{4}$ \\
$\bar{v}_{\mu}$ & 1.6 & $2.1 \times 10^{18}$ & 8.6 & $3.5 \times 10^{16}$ & 26 & $8.6 \times 10^{5}$ \\
$\bar{v}_{e}$ & 4.7 & $1.8 \times 10^{17}$ & 21.6 & $5.2 \times 10^{15}$ & 38 & $2.2 \times 10^{5}$ \\
$\bar{v}_{\tau}$ & 8.1 & $1.1 \times 10^{16}$ & 30.7 & $4.7 \times 10^{14}$ & 58 & $1.9 \times 10^{4}$ \\
\hline
\end{tabular}

Table 1: Integrated neutrino yield for $2 \times 10^{20}$ p.o.t. and corresponding mean energies for the different neutrino flavours at the Beam Dump (right column), at the Neutrino Detector (central column) and their charged current interactions (right column).

of tab. 1. All the results have been obtained using a Geant 4 based simulation of the facility. It is important to note that almost $26000 v_{\tau}$ and $19000 \bar{v}_{\tau}$ charged current interactions are expected.

The full expression for the neutrino differential Deep Inelastic Scattering (DIS) cross section on a nucleon is:

$$
\begin{aligned}
\frac{d^{2} \sigma^{v(\bar{v})}}{d x d y} & =\frac{G_{F}^{2} M E_{v}}{\pi\left(1+Q^{2} / M_{W}^{2}\right)^{2}}\left\{\left(y^{2} x+\frac{m_{\tau}^{2} y}{2 E_{v} M}\right) F_{1}+\left[\left(1-\frac{m_{\tau}^{2}}{4 E_{v}^{2}}\right)-\left(1+\frac{M x}{2 E_{v}}\right)\right] F_{2}\right. \\
& \left. \pm\left[x y\left(1-\frac{y}{2}\right)-\frac{m_{\tau}^{2} y}{4 E_{v} M}\right] F_{3}+\frac{m_{\tau}^{2}\left(m_{\tau}^{2}+Q^{2}\right)}{4 E_{v}^{2} M^{2} x} F_{4}-\frac{m_{\tau}^{2}}{E_{v} M} F_{5}\right\},
\end{aligned}
$$

where $E_{v}$ is the initial neutrino energy and $M$ and $m_{\tau}$ are the mass of the nucleon and the mass of the $\tau$ lepton respectively. It is expressed in terms of five structure functions $F_{i}, i \in 1, . ., 5$, which in the Parton Model can be interpreted as a measure of the partonic structure of hadrons. The positive sign multiplying $F_{3}$ applies to neutrino scattering while the negative sign applies to antineutrinos.

In SHiP, the possibility to perform studies of DIS of $v_{\tau}\left(\bar{v}_{\tau}\right)$ on nuclei opens the way to the measurement of the structure functions $F_{4}$ and $F_{5}$, that, unlike the others, are proportional to the ratio $m_{l}^{2} / E_{l}$, negligible in muon (and electron) neutrino interactions being $m_{l}$ the associated charged lepton mass. At leading order, in the limit of massless quarks and target hadrons, $F_{4}=0$ and $2 x F_{5}=F_{2}$, where $x$ is the Bjorken- $x$ variable [8]. Calculations at NLO show that the $F_{4}$ contribution to this cross-section is about $1 \%$ [9]. As a matter of fact, a non null value of $F_{4}$ and $F_{5}$ affects the DIS cross-section which increases with respect to the hypothesis of both $F_{4}$ and $F_{5}$ equal to zero, especially at lower neutrino energies. The variable $r$, defined as the ratio between the cross section in the NLO and LO hypothesis, reflects these discrepancies.

In fig. 4 a the variable $r$ as a function of the neutrino energy is displayed for $\bar{v}_{\tau}$. Simulations have shown that SHiP can probe a non-zero value of $F_{4}$ and $F_{5}$ by observing more than 5000 expected events with $E<38 \mathrm{GeV}$ if only the $\bar{v}_{\tau}$ dataset is considered.

Thanks to the large flux of $v_{e}$ and $v_{\mu}$ reaching and interacting in the neutrino target, not only studies of $v_{\tau}$ physics can be performed. The strange-quark content of the nucleon can be measured by means of the charmed hadron production in anti-neutrino interactions. In 5 years running, more 
than $10^{5}$ neutrino induced charmed hadrons are expected. The use of nuclear emulsions allows to identify the charmed hadrons on topological basis distinguishing both the neutrino interaction vertex and the vertex produced by the charmed hadron decay, occurring after about one millimetre. Therefore, all the decay channels of the charmed hadrons turn out to be accessible without any kinematical cut. Not only di-lepton events have thus to be taken into account making the available statistics exceed by more than one order of magnitude that of previous experiments.

A simulated SHiP data sample normalized to the expected statistics was used to estimate the distribution of $v_{\mu}\left(\bar{v}_{\mu}\right)$ interactions with charm production. The potential impact of simulated charm data on the knowledge of the nucleon strangeness was assessed by adding them to the NNPDF3.0 NNLO fit [10].

In fig. $4 \mathrm{~b}$, the uncertainty on the knowledge of parton distribution functions of $s+\bar{s}$ is shown. The blue areas represent the current NNLO estimates [4], while the red areas take into account the information obtained in 5 year run of SHiP, leading to an improvement in the knowledge of parton densities of almost a factor two gain in the $x$ range between 0.03 and 0.35 , a region complementary to that explored by ATLAS and CMS which are sensitive at lower values of $x$.

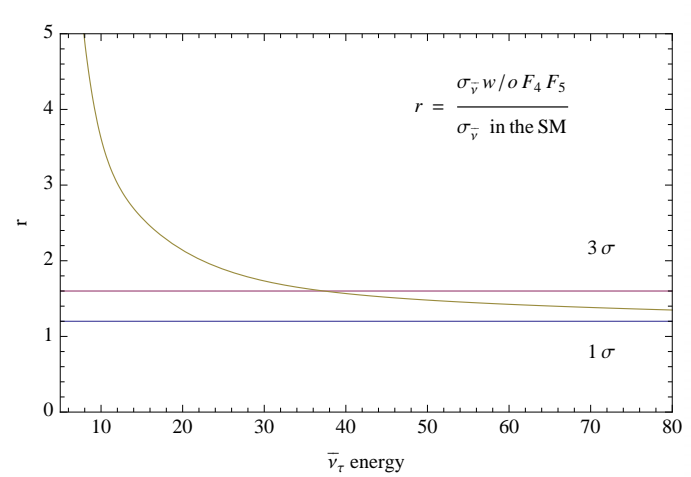

(a)

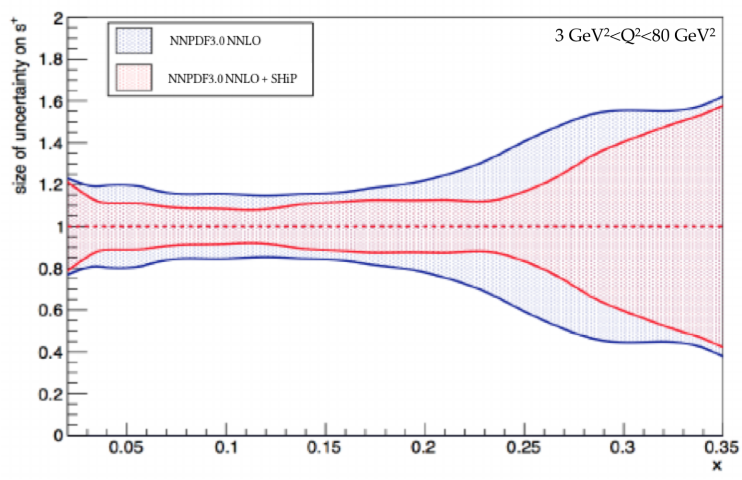

(b)

Figure 4: (a) Energy dependence of the ratio $r$ between the DIS cross-section in the $F_{4}=F_{5}=0$ hypothesis and in the SM hypothesis for $\bar{v}_{\tau}$. (b) Present (in blue) and future (in red) knowledge of parton distribution functions of $s+\bar{s}$ for x spanning from 0.03 to 0.35 in a $Q^{2}$ region between 3 $\mathrm{Gev}^{2}$ and $80 \mathrm{Gev}^{2}$.

Experiments performing direct dark matter (DM) searches are still not sensitive to DM particles with masses of $\mathrm{O}(\mathrm{GeV})$ or lighter. This mass region can be explored by accelerator experiments, where the target recoils gain enough energy to be detected. In a proton dump, light DM particles $(\chi)$ can be produced via the decay of the dark photon ( [11-14]) and the neutrino detector is well suited to identify them through the scattering off electrons. Background sources for this search are neutral current $v_{\mu}$ and $v_{e}$ scattering on electrons, and charged current elastic, resonant and deep inelastic $v_{e}$ scattering off nuclei. The main variables to separate signal from background are the electron energy and the angle with respect to the neutrino direction and the number of detectable particles at the neutrino interaction vertex. Preliminary studies have shown that the sensitivity that the SHiP neutrino detector can achieve considerably extends the reach of previous experiments. 


\section{References}

[1] ATLAS Collaboration, Phys.Lett., B716, 129 (2012).

[2] CMS Collaboration, Phys.Lett., B716, 3061 (2012).

[3] SHIP Collaboration, CERN-SPSC-2015-016, SPSC-P-350, arXiv:1504.04956 [physics.ins-det], (2015).

[4] SHIP Collaboration, CERN-SPSC-2015-017, arXiv:1504.04855 [hep-ph], (2015).

[5] C. Lourenco and H. Wohri, Phys.Rept. 433, 127-180, (2006).

[6] H. Abramowicz et al., JHEP. 1309, 058, (2013).

[7] K. Olive et al., Review of Particle Physics, Chin.Phys. C38, 090001, (2014)

[8] C. H. Albright and C. Jarlskog, Nucl. Phys., B84, 467 (1975) .

[9] M. H. Reno, Phys. Rev., D74, 033001 (2006) .

[10] R. D. Ball et al., JHEP, 1504, 040 (2015).

[11] B. Batell, M. Pospelov, and A. Ritz, Phys.Rev., D80, 095024 (2009).

[12] Y. Kahn, G. Krnjaic, J. Thaler, and M. Toups, Phys.Rev., D91(5), 055006 (2015).

[13] P. deNiverville, D. McKeen, and A. Ritz, Phys.Rev., D86, 035022 (2012).

[14] B. Batell, P. deNiverville, D. McKeen, M. Pospelov, and A. Ritz, Phys.Rev., D90(11), 115014 (2014). 